\title{
Meson spectra in a gluon condensate background
}

\author{
Yumi Ko $*$, Bum-Hoon Lee ${ }^{a \ell t}$, and Chanyong Park ${ }^{a t}$ \\ a Center for Quantum Spacetime (CQUeST), Sogang University, Seoul 121-742, Korea \\ ${ }^{b}$ Department of Physics, Sogang University, Seoul 121-742, Korea
}

\begin{abstract}
We study the spectra of light mesons as well as the dissociation of a quarkonium and monopole-anti monopole bound state in a gluon condensate background. In order to describe the confining phase we introduce an IR cutoff in two ways, namely the hard wall and the braneless approaches. We find that the meson spectra strongly depend on the gluon condensate in the braneless approach, while they do not in the hard wall model. The latter result appears to be a more probable feature of QCD.
\end{abstract}

*e-mail : koyumi@sogang.ac.kr

${ }^{\dagger}$ e-mail : bhl@sogang.ac.kr

${ }^{\ddagger}$ e-mail : cyong21@sogang.ac.kr 


\section{Contents}

\begin{tabular}{ll|l}
1 & Introduction & 1
\end{tabular}

2 Gluon condensate background $\quad 2$

3 Hard wall approach 4

3.1 Vector meson . . . . . . . . . . . . . . . . . . 5

3.2 Chiral condensate . . . . . . . . . . . . . . . . . . . 6

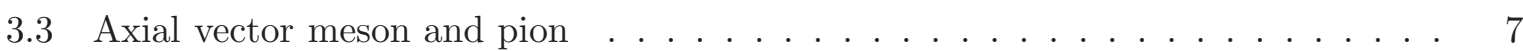

3.4 Dissociation of a quarkonium and a monopole bound state . . . . . . . . . 7

4 Braneless approach $\quad 10$

5 Summary and Discussion $\quad 12$

\section{Introduction}

Low energy QCD is an important and challenging field to study in spite of the difficulties in dealing with its nonperturbative nature. Recently, there have been many attempts, termed holographic QCD, to understand such strongly interacting systems based on the AdS/CFT correspondence 1, 2, 3, 4, 5, 6, 7, 8, 9, 10, 11, 12. The holographic idea was applied to construct a dual gravity model for QCD incorporating chiral symmetry breaking in Refs. [5, 6, 7, 11, 12. By introducing an IR cutoff or a dilaton potential in the AdS space to describe the quark confinement, the masses of the various mesons and decay constants were investigated in these works.

In the confining phase there exist important vacuum quantities such as the baryon number density, the chiral condensate and gluon condensate which can affect these physical properties such as the meson spectrum. Therefore it is of interest to properly implement these effects in the holographic QCD model. To do so, we should find the dual geometry in the confining phase, include these vacuum effects and investigate how they change the physical quantities such as the mass spectrum, the deconfinement temperature, and other relevant quantities.

For the case of a finite baryon density, the thermally charged AdS space was proposed as the dual geometry of the hadronic phase with a finite baryonic chemical potential or number density [13. In this background, the light meson and quarkonium spectra and the deconfinement phase transition were studied in Refs. 14, 15, 16. The chiral condensation is dual to a massive scalar field in the bulk. In Ref. [17] the first correction of the massive scalar field to the Hawking-Page transition temperature was investigated in a background without backreaction of the massive scalar field. As the dual geometry containing the chiral condensate is still not known, it would be an interesting problem in itself to find the full solution including the backreaction of the 
scalar field. In the case with a specific scalar potential [18], meson spectra has been studied in a background containing a backreaction of the massive scalar field. On the other hand, the dual geometry including the gluon condensate can be obtained by analytically solving a gravity theory coupled to a dilaton, i.e. a massless scalar field [19, 20, 21, 22, 23. Usually, there are two types of solutions, the dilaton wall solution and the dilaton black hole solution. These correspond to the confining and deconfining backgrounds, respectively. In the deconfining background the effect of the gluon condensate on the quarkonium spectrum as well as the deconfinement phase transition have both been already investigated in Ref. [24].

In this work, we study the effects of gluon condensation on the light meson spectra and the dissociation of the quarkonium and monopole bound state in a confining background. As we will see, the dilaton wall solution becomes divergent at $z=z_{c}$. However, it is known as a "good singularity" which does not appear in the action because the divergent terms coming from the curvature scalar and the dilaton kinetic term are exactly canceling each other. In order to calculate the mass spectrum of mesons in a confining background, we need to take into account an IR cutoff. For this, we can either take the singular point $z_{c}$ as an intrinsic IR cutoff or insert by hand a hard wall at $z=z_{I R}$ in front of $z_{c}$. We refer to the first procedure as the "braneless" approach, and the second as the "hard wall" approach.

In QCD, the effect of the baryon density and the chiral condensate on physical properties of quarks are expected to be stronger than the gluon condensation effect. However, the gluon condensate background is of theoretical interest because it may be solved analytically. Moreover, this work may shed further light on studies of the chiral condensate dependence of the meson spectra in holographic QCD, and on investigations of superconductivity in AdS/CMT. Thus it is meaningful to study the behavior of physical quantities depending on the gluon condensate. To describe more realistic phenomena, the model should be improved by incorporating the effect of chiral condensates and the baryon number density in the geometry and should be studied elsewhere.

We organize our paper as follows: in Sec. 2, we provide a review of gluon condensation and its dual geometry. Next we consider the mass spectrum of various types of mesons, in the presence (Sec. 3) and absence (Sec 4.) of a hard wall as an IR cutoff. We will call these two approaches the hard wall approach and the braneless approach, respectively. Finally, we discuss our results in sec. 5 .

\section{Gluon condensate background}

In this section, we will shortly review gluon condensation and its dual geometry [19, 20, 21, 22. In holography, the gluon condensation is dual to the dilaton field on the gravity side. Thus we

consider a five-dimensional Euclidean gravity action with a cosmological constant, $\Lambda=-\frac{6}{R^{2}}$, 
and a dilaton field [22, 20]

$$
S=\frac{1}{2 \kappa^{2}} \int d^{5} x \sqrt{G}\left(-\mathcal{R}+2 \Lambda+\frac{1}{2} \partial_{M} \phi \partial^{M} \phi\right) .
$$

Here $\kappa^{2}$ is the five-dimensional Newton constant and $R$ is a curvature radius. The Einstein equation and the equation of motion for the scalar field read

$$
\begin{aligned}
\mathcal{R}_{M N}-\frac{1}{2} G_{M N} \mathcal{R}+G_{M N} \Lambda & =\frac{\kappa^{2}}{2}\left[\partial_{M} \phi \partial_{N} \phi-\frac{1}{2} G_{M N} \partial_{P} \phi \partial^{P} \phi\right], \\
0 & =\frac{1}{\sqrt{G}} \partial_{M} \sqrt{G} G^{M N} \partial_{N} \phi .
\end{aligned}
$$

As shown in Ref. [24], there exist two solutions. One is a dilaton wall solution, i.e. a deformation of AdS spacetime. It corresponds to the confining phase with gluon condensation. The other is a dilaton black hole solution, i.e. a deformation of the Schwarzschild-type AdS black hole with a naked singularity at the horizon. The naked singularity gives rise to some problems, e.g. a proper definition of the Hawking temperature. As the aim of this paper is to study the meson spectra depending on the gluon condensation in the confining phase, we will only focus on the dilaton wall solution which forms as follows:

$$
\begin{aligned}
d s^{2} & =\frac{R^{2}}{z^{2}}\left(\sqrt{1-c^{2} z^{8}} \delta_{\mu \nu} d x^{\mu} d x^{\nu}+d z^{2}\right), \\
\phi(z) & =\phi_{0}+\sqrt{\frac{3}{2}} \log \left(\frac{1+c z^{4}}{1-c z^{4}}\right),
\end{aligned}
$$

where $\phi_{0}$ and $c$ are integration constants.

Besides the gluon condensation, there are other important effects in the confining phase such as the chiral condensation and the quark density effects. In both cases, the number of degrees of freedom is proportional to $N_{c} N_{f}$ while $N_{c}^{2}$ for the gluon condensation. Thus, in the limit $N_{c} \gg N_{f}$ in confining phase, the effect of gluon condensation on the physical properties becomes dominant. This regime is what we are interested in this paper. It would also be interesting to investigate the effect of chiral condensation for $N_{c} \sim N_{f}$, and will be left for future work.

In order to describe the confining behavior of the dual gauge theory, we need to introduce an IR cutoff. For this purpose, we choose two possible ways, namely the "hard wall approach" and the "braneless approach."

In the hard wall approach, we insert a hard wall as an IR cutoff in front of the singular point $z_{c} \equiv \frac{1}{c^{1 / 4}}$ by hand. In this case, we do not need to worry about the singularity as it is screened by the IR cutoff. On the other hand, the singular point $z_{c}$ itself can be regarded as an IR cutoff, which is the principle of the braneless approach. Its merit is that we do not need to introduce an IR cutoff by hand. At a glance, one may wonder whether the logarithmic divergence of the scalar field in Eq. (3) at $z_{c}$ causes some problems for the calculation of the meson spectra. However, as will be shown later, the logarithmic divergence is removed as the metric component vanishes at $z_{c}$. 
Near the boundary when $z \rightarrow 0$, the perturbative expansion of the dilaton field becomes

$$
\phi=\phi_{0}+\sqrt{6} \frac{z^{4}}{z_{c}^{4}}+\mathcal{O}\left(z^{8}\right) .
$$

According to the AdS/CFT correspondence, the constant term, $\phi_{0}$, corresponds to a source for the gluon condensation operator $\operatorname{Tr} G^{2}$ and the coefficient of the normalizable mode gives the gluon condensate which is 22$]$

$$
\left\langle\operatorname{Tr} G^{2}\right\rangle=\frac{8 \sqrt{3\left(N_{c}^{2}-1\right)}}{\pi} \frac{1}{z_{c}^{4}}
$$

where $\frac{1}{\kappa^{2}}=\frac{4\left(N_{c}^{2}-1\right)}{\pi^{2} R^{3}}$ is used. To consider the meson spectra on the gluon condensate background, we should turn on several bulk field fluctuations on the dilaton wall background. The action of these fluctuations is given by [5, 7]

$$
\Delta S=\int d^{5} x \sqrt{G} \operatorname{Tr}\left[|D X|^{2}-\frac{3}{R^{2}}|X|^{2}+\frac{1}{4 g_{5}^{2}}\left(F_{L}^{2}+F_{R}^{2}\right)\right],
$$

with

$$
\frac{1}{g_{5}^{2}}=\frac{N_{c}}{12 \pi^{2} R}
$$

where $R$ and $N_{c}$ are needed for the correct dimensionality and proper $1 / N_{c}$ suppression, respectively. Note that $D$ is a covariant derivative, $D_{M} X=\partial_{M} X-i A_{M}^{(L)} X+i X A_{M}^{(R)}$ and $F_{M N}^{(L, R)}=\partial_{M} A_{N}^{(L, R)}-\partial_{N} A_{M}^{(L, R)}-i\left[A_{M}^{(L, R)}, A_{N}^{(L, R)}\right]$, where the combinations of $A_{M}^{L}$ and $A_{M}^{R}$

$$
\begin{aligned}
V_{M} & =\frac{1}{2}\left(A_{M}^{L}+A_{M}^{R}\right), \\
A_{M} & =\frac{1}{2}\left(A_{M}^{L}-A_{M}^{R}\right),
\end{aligned}
$$

correspond to the vector meson or axial vector meson, respectively. The complex scalar field $X$ transforms in the bifundamental representation under the flavor symmetry group, $S U\left(N_{f}\right)_{L} \times$ $S U\left(N_{f}\right)_{R}$, which can be parameterized as

$$
X=\frac{v}{2} e^{2 i \pi}
$$

where the background field $v$ and fluctuation $\pi$ corresponds to the quark mass (or chiral condensate) and pseudoscalar meson.

\section{$3 \quad$ Hard wall approach}

In the gluon condensate background as shown in the previous section, there exists a singular point, which can be considered as an IR cutoff. We will call this set-up a braneless approach which will be investigated in the next section. In this section, we will consider a hard wall approach, in which we insert a new IR cutoff by hand in front of the geometric singular point. 
There are several motivations why we introduce the new IR cutoff. The first one is related to the generalization of this model. For the massless scalar case, the on-shell gravity action is finite since the divergences from the curvature and the kinetic term of the scalar field cancel each other exactly. So the free energy of dual QCD corresponding to on-shell bulk gravity action is finite. If we add a mass or potential term for the scalar field, the on-shell gravity action diverges if there exists a geometrical cutoff. Therefore, the dual free energy is not well defined. This fact implies that the geometrical cutoff can not be generally considered as a proper IR cutoff. One way to cure this problem is to introduce another IR cutoff screening the geometrical singular point. The second one is how to impose the IR boundary condition at the geometrical cutoff where the scalar field and curvature diverge. Actually, the IR boundary condition plays an important role to determine the relation between various parameters. If we insert the new IR cutoff, we can easily impose the IR boundary condition although it still remains to find a physical IR boundary condition explaining the parameter relations in QCD.

In this paper, we will investigate the light meson and quarkonium spectra in these two approaches. For comparing our results with those in Ref. [5], we will start with the hard wall approach, in which we insert an IR brane (or hard wall) in front of the singular point $z_{c}$. If the position of the hard wall is $z=z_{I R}<z_{c}$, the range of the $z$-coordinate in the bulk space is limited to $0 \leq z \leq z_{I R}$. In this case the singular point lies outside the bulk, and we do not need to worry about the singularity. In this paper we choose $z_{I R}=(323 \mathrm{MeV})^{-1}$ because it generates the correct $\rho$-meson mass in the original hard wall model [5].

\subsection{Vector meson}

Following the AdS/CFT correspondence, the bulk gauge field fluctuations correspond to the meson in holographic QCD. In this section, we will investigate the vector meson spectrum by turning on the vector gauge field, which does not mix with the scalar and axial gauge field. In the axial gauge $V_{z}=0$, the equations of motion for the vector gauge field $V_{i}(i=1,2,3)$ become

$$
0=\frac{1}{\sqrt{G}} \partial_{M} \sqrt{G} G^{M P} G^{i j} \partial_{P} V_{i}
$$

where $M$ and $P$ are five-dimensional indices. Using the ansatz,

$$
V_{i}=\int \frac{d^{4} k}{(2 \pi)^{4}} e^{-i \omega_{n} t+i \vec{p}_{n} \vec{x}} V_{i}^{(n)}(z)
$$

where $n$ implies the $n$-th excitation mode, the above equations are reduced to

$$
0=\partial_{z}^{2} V_{i}^{(n)}-\frac{1+3 c^{2} z^{8}}{z\left(1-c^{2} z^{8}\right)} \partial_{z} V_{i}^{(n)}+\frac{m_{n}^{2}}{\sqrt{1-c^{2} z^{8}}} V_{i}^{(n)}
$$

Here, $m_{n}^{2}=w_{n}^{2}-\vec{p}_{n}^{2}$ is a mass squared of the $n$-th excited meson state. Especially, the first excited state is called $\rho$-meson, whose mass is denoted by $m_{\rho}$. To obtain the vector meson mass, we impose the Dirichlet boundary condition $\left(V_{i}^{(n)}=0\right)$ at the UV fixed point $(z=0)$ and the 
Neumann boundary condition $\left(\partial_{z} V_{i}^{(n)}=0\right)$ at the IR cutoff $\left(z=z_{I R}\right)$. Since the range of $z$ is finite, the boundary conditions are satisfied only at some discrete values of $m_{\rho}$ for $n=1$. We find the values of $m_{\rho}$ depending on gluon condensation numerically, as listed in Table 1 . We see that as the gluon condensation increases, the vector meson mass decreases.

\begin{tabular}{|c||c|c|c|c|}
\hline$z_{c}(1 / \mathrm{GeV})$ & $\left\langle\operatorname{Tr} G^{2}\right\rangle\left(\mathrm{GeV}^{4}\right)$ & $m_{\rho}(\mathrm{GeV})$ & $m_{A}(\mathrm{GeV})$ & $m_{\pi}(\mathrm{GeV})$ \\
\hline \hline$\infty$ & 0 & 0.7767 & 1.3582 & 0.13961 \\
\hline $1 / 0.176$ & 0.012 & 0.7767 & 1.3583 & 0.13961 \\
\hline $1 / 0.200$ & 0.020 & 0.7767 & 1.3584 & 0.13961 \\
\hline $1 / 0.250$ & 0.049 & 0.7762 & 1.3589 & 0.13964 \\
\hline $1 / 0.280$ & 0.077 & 0.7755 & 1.3599 & 0.13970 \\
\hline $1 / 0.320$ & 0.131 & 0.7724 & 1.3612 & 0.13999 \\
\hline
\end{tabular}

Table 1 : Various meson masses depending on the gluon condensation in the hard wall approach.

\subsection{Chiral condensate}

The chiral condensation is obtained by solving for $v(z)$, i.e. the expectation value of the complex scalar field $X$. From the action of Eq. (6), the equation of motion for $v(z)$ is given by

$$
\begin{aligned}
0 & =\partial_{z}\left[\sqrt{G} g^{z z} \partial_{z} v\right]+\frac{3}{R^{2}} \sqrt{G} v \\
& =\partial_{z}^{2} v-\frac{3+5 c^{2} z^{8}}{z\left(1-c^{2} z^{8}\right)} \partial_{z} v+\frac{3}{z^{2}} v .
\end{aligned}
$$

Its exact solution is

$$
v=A z_{2} F_{1}\left[\frac{1}{4}, \frac{1}{2}, \frac{3}{4}, c^{2} z^{8}\right]+B z^{3}{ }_{2} F_{1}\left[\frac{1}{2}, \frac{3}{4}, \frac{5}{4}, c^{2} z^{8}\right],
$$

where $A$ and $B$ are integration constants. Near the UV cutoff $(z \rightarrow 0), v(z)$ perturbatively expands as

$$
v=A z+B z^{3}+\mathcal{O}\left(z^{9}\right),
$$

and accords with the solution on the pure AdS background. Now we can identify the coefficient of the non-normalizable mode to the current quark mass $m_{q}$ as

$$
A=m_{q},
$$

where $R$ is set to one for simplicity, and the coefficient of the normalizable mode is related to the chiral condensate as

$$
B=\sigma \equiv\langle\bar{q} q\rangle .
$$

Notice that each term on the right hand side of Eq. (14) has a logarithmic divergence at $z=z_{c}$. As we expand each term near $z_{c}$, we encounter the divergence which reads

$$
\begin{aligned}
& z_{2} F_{1}\left[\frac{1}{4}, \frac{1}{2}, \frac{3}{4}, \frac{z^{8}}{z_{c}^{8}}\right]=-0.19069 z_{c}\left(\log \frac{\epsilon}{z_{c}}-2.95709\right)+\mathcal{O}(\epsilon), \\
& z^{3}{ }_{2} F_{1}\left[\frac{1}{2}, \frac{3}{4}, \frac{5}{4}, \frac{z^{8}}{z_{c}^{8}}\right]=-0.41731 z_{c}^{3}\left(\log \frac{\epsilon}{z_{c}}+0.184502\right)+\mathcal{O}(\epsilon),
\end{aligned}
$$


where $\epsilon=z-z_{c}$. Even though the value of $v$ becomes singular at $z=z_{c}$, the logarithmic divergence does not affect our calculation as the range of $z$ is restricted to $0 \leq z \leq z_{I R}$ with $z_{I R}<z_{c}$

\subsection{Axial vector meson and pion}

Let us make the following ansatz for the axial gauge field,

$$
\begin{aligned}
A_{\mu} & =\int d^{4} k e^{i q x}\left(\bar{A}_{\mu}+\partial_{\mu} \varphi\right), \\
\pi & =\int d^{4} k e^{i q x} \pi_{q},
\end{aligned}
$$

where $\bar{A}$ means a transverse gauge field in the bulk satisfying $\partial_{\mu} A^{\mu}=0$ and $\varphi$ a gauge transformation. The equations of motion for the axial gauge field and the pseudoscalar fields are

$$
\begin{aligned}
0 & =\partial_{z}\left(\frac{\sqrt{1-z^{8} / z_{c}^{8}}}{z} \partial_{z} \bar{A}_{\mu}\right)+\frac{m_{A}^{2}}{z} \bar{A}_{\mu}-\frac{g_{5}^{2} \Gamma(z)}{z^{3}} \bar{A}_{\mu}, \\
0 & =\partial_{z}\left(\frac{\sqrt{1-z^{8} / z_{c}^{8}}}{z} \partial_{z} \varphi\right)+\frac{g_{5}^{2} \Gamma(z)}{z^{3}}\left(\pi_{q}-\varphi\right), \\
0 & =-m_{\pi}^{2} \partial_{z} \varphi+\frac{g_{5}^{2} \Gamma(z)}{z^{2}} \partial_{z} \pi_{q},
\end{aligned}
$$

where $\Gamma(z)=v^{2} \sqrt{1-z^{8} / z_{c}^{8}}$ and we set $R=1$. Since the first equation for the axial vector meson is decoupled from the two other ones, we can numerically solve it and determine the mass of the axial vector meson. We again impose a Dirichlet boundary condition $\left(\bar{A}_{\mu}=0\right)$ at the UV fixed point, $z=0$, and a Neumann boundary condition $\left(\partial_{z} \bar{A}_{\mu}=0\right)$ at the IR cutoff, $z=z_{I R}$. In Table 1, we show the masses of the first excited axial vector meson, varying values of the gluon condensation. Here, we used $m_{q}=2.29 \mathrm{MeV}$ and $\sigma=(327 \mathrm{MeV})^{3}$ obtained in Ref. [5] to compare our results with the ones of the original hard wall model.

The pion mass can be obtained by solving the last two coupled equations in Eq. (20). We choose three boundary conditions, $\varphi=0$ and $\pi_{q}=0$ at the boundary, and $\partial_{z} \varphi=0$ at the IR cutoff, $z=z_{I R}$. See also Table 1 for the mass of the pion and mass of the first excited pseudoscalar meson as functions of the gluon condensate.

We find that the gluon condensate dependence of the various meson masses is rather weak. However, from a qualitative point of view, increasing the gluon condensate leads to an increase of the axial vector meson mass and the pion mass and to a decrease of the vector meson mass.

\subsection{Dissociation of a quarkonium and a monopole bound state}

So far, we have studied mass spectra for various light mesons depending on the gluon condensate. Let us now consider the quarkonium and the monopole-anti monopole bound state, described by a fundamental open string and a D1-string respectively. In this section, we investigate the gluon condensate dependence of their dissociation length in the confining phase. 
First, we consider the quarkonium composed of two heavy quarks. The fundamental string action in the gluon condensated background [19, 22, 20] is given by

$$
S_{F 1}=\frac{1}{2 \pi \alpha^{\prime}} \int d^{2} \sigma e^{\phi / 2} \sqrt{\operatorname{det} h_{\alpha \beta}}
$$

where $h_{\alpha \beta}$ is the induced metric on the string worldsheet. For simplicity, we take

$$
\frac{R^{2}}{2 \pi \alpha^{\prime}}=1 \quad \text { and } \quad \phi_{0}=0
$$

At first we consider the string configuration connecting two heavy quarks with the following ansatz

$$
\tau=t, \quad \sigma=x^{1} \equiv x \quad \text { and } \quad z=z(x),
$$

the fundamental string action in the background of Eq. (3) is then reduced to

$$
S_{F 1}=\int_{-T / 2}^{T / 2} d t \int_{-r / 2}^{r / 2} d x \frac{\Delta}{z^{2}} \sqrt{1-c^{2} z^{8}+\sqrt{1-c^{2} z^{8}} z^{\prime 2}},
$$

where $r$ is the distance between the quark and the anti-quark in the $x$ direction and $\Delta$ is given by

$$
\Delta=\left(\frac{1+c z^{4}}{1-c z^{4}}\right)^{\sqrt{3 / 8}}
$$

Then the conserved Hamiltonian of this system can be obtained as

$$
H=-\frac{\Delta}{z^{2}} \frac{1-c^{2} z^{8}}{\sqrt{1-c^{2} z^{8}+\sqrt{1-c^{2} z^{8} z^{\prime 2}}}} .
$$

If there exists a point $z_{0}$ where $\left.\frac{\partial z}{\partial x}\right|_{z=z_{0}}$ becomes zero, the Hamiltonian at this point becomes

$$
H=-\frac{\Delta_{0}}{z_{0}^{2}} \sqrt{1-c^{2} z_{0}^{8}}
$$

where $\Delta_{0}$ is the value of $\Delta$ at $z=z_{0}$. Then the conservation law leads us to find the relation between $r$ and $z_{0}$ from Eq. (26) and Eq. (27) as

$$
r=2 \int_{0}^{z_{0}} d z \frac{z^{2}}{z_{0}^{2}} \frac{\sqrt{1-c^{2} z_{0}^{8}}}{\left(1-c^{2} z^{8}\right)^{1 / 4}} \frac{1}{\sqrt{\frac{\Delta^{2}}{\Delta_{0}^{2}}\left(1-c^{2} z^{8}\right)-\frac{z^{4}}{z_{0}^{4}}\left(1-c^{2} z_{0}^{8}\right)}}
$$

In addition, the quarkonium free energy proportional to the string on-shell action becomes

$$
E_{q}=2 \int_{0}^{z_{0}} d z \frac{\Delta^{2}}{z^{2} \Delta_{0}} \frac{\left(1-c^{2} z^{8}\right)^{3 / 4}}{\sqrt{\frac{\Delta^{2}}{\Delta_{0}^{2}}\left(1-c^{2} z^{8}\right)-\frac{z^{4}}{z_{0}^{4}}\left(1-c^{2} z_{0}^{8}\right)}} .
$$

Since we consider the static string configuration in which the kinetic energy of quarks vanishes, the above free energy can be interpreted as a sum of the quark masses and the interaction energy between two heavy quarks. Notice that the quarkonium free energy in Eq. (29) diverges at the 


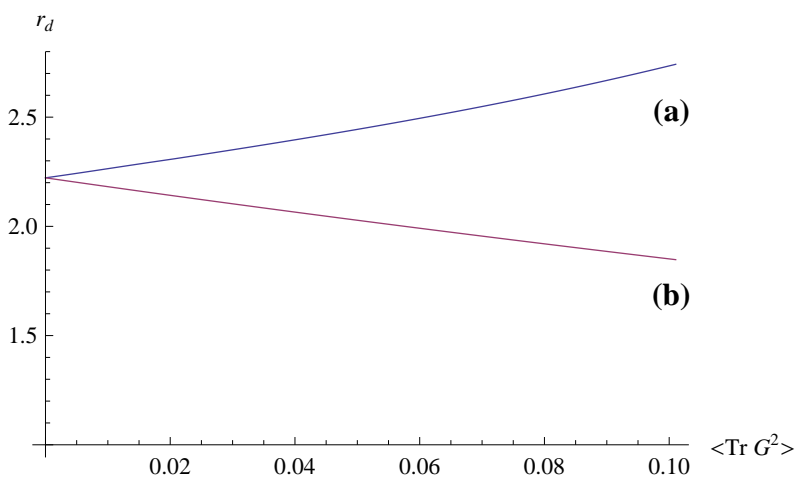

Figure 1: Dissociation length $r_{d}$ in the unit of $\mathrm{GeV}^{-1}$, depending on the gluon condensation in the case of (a) quarkonium and (b) the bound state of monopole-anti monopole in the hard wall approach.

boundary $z=0$. This is due to the infinite mass of a heavy quark in the hard wall model. To understand this, we consider a straight string configuration. Under the following ansatz

$$
\tau=t, \quad \sigma=z \quad \text { and } \quad x_{1}=\text { const }
$$

the free energy of two straight strings extended from 0 to $z_{I R}$ in $z$-direction, which corresponds to a mass of two heavy quarks in QCD side, is reduced to

$$
E_{m}=2 \int_{0}^{z_{I R}} d z \frac{\Delta\left(1-c^{2} z^{8}\right)^{1 / 4}}{z^{2}} .
$$

From this, we can see that the heavy quark in this model has an infinite mass. Therefore to obtain a finite interaction energy we should calculate the free energy difference of two string configurations, which we call effective potential

$$
V_{F 1}=E_{q}-E_{m}
$$

If the above effective potential is bigger than the mass of two light quarks, the quarkonium should be broken to two heavy-light mesons. Furthermore, since the mass of a light quark is very small we can ignore the effect of the light quark mass. Then, $V_{F 1}<0$ (or $V_{F 1}>0$ ) implies that the quarkonium is more stable (or unstable) than two heavy-light meson. So the interquark distance $r$ at $V_{F 1}=0$ can be interpreted as a dissociation length. Figure 1 (a) shows the dissociation length of quarkonium depending on the gluon condensation.

For describing the bound state of monopole and anti monopole, we consider a D1-string instead of a fundamental string, whose action is given by [26, 27]

$$
\begin{aligned}
S_{D 1} & =\int d^{2} \sigma e^{-\phi} \sqrt{\operatorname{det} G^{M N} \partial_{\alpha} X^{M} \partial_{\beta} X^{N}} \\
& =\int_{-T / 2}^{T / 2} d t \int_{-r / 2}^{r / 2} d x \frac{1}{z^{2} \Delta} \sqrt{1-c^{2} z^{8}+\sqrt{1-c^{2} z^{8}} z^{\prime 2}}
\end{aligned}
$$


where Eq. (22) is used. Following the same procedure used in the fundamental string case, the interquark distance $r$ and the free energy difference $E_{D 1}$ between the monopole and the anti-monopole can be written as functions of $z_{0}$ as follows:

$$
\begin{aligned}
r=2 \int_{0}^{z_{0}} d z \frac{z^{2}}{z_{0}^{2}} \frac{\sqrt{1-c^{2} z_{0}^{8}}}{\left(1-c^{2} z^{8}\right)^{1 / 4}} \frac{1}{\sqrt{\frac{\Delta_{0}^{2}}{\Delta^{2}}\left(1-c^{2} z^{8}\right)-\frac{z^{4}}{z_{0}^{4}}\left(1-c^{2} z_{0}^{8}\right)}}, \\
V_{D 1=}=2 \int_{0}^{z_{0}} d z \frac{\Delta_{0}}{z^{2} \Delta^{2}} \frac{\left(1-c^{2} z^{8}\right)^{3 / 4}}{\sqrt{\frac{\Delta_{0}^{2}}{\Delta^{2}}\left(1-c^{2} z^{8}\right)-\frac{z^{4}}{z_{0}^{4}}\left(1-c^{2} z_{0}^{8}\right)}} \\
-2 \int_{0}^{z_{I R}} d z \frac{\left(1-c^{2} z^{8}\right)^{1 / 4}}{z^{2} \Delta} .
\end{aligned}
$$

Then the dissociation length of the monopole-anti monopole bound state depending on the gluon condensation can be found numerically, see Figure 1 (b). We find that the dissociation length of the quarkonium becomes large as the gluon condensation increases, which implies that it is difficult to dissociate the quarkonium due to the gluon condensation. On the contrary, the monopole-anti monopole bound state can be dissociated more easily as the gluon condensation increases.

\section{Braneless approach}

As mentioned previously, we can take into account another approach to study hadron physics in the confining phase, so called braneless approach. In this case, since we use the intrinsic singular point $z_{c}$ as an IR cutoff, we do not need to introduce an artificial IR bound by hand. Taking this to our advantage, we will now study the meson spectra in the braneless approach.

Note that all equations of motion for meson spectra are the same as the ones in the hard wall case, and the only difference is that the IR boundary position in the braneless approach is given by $z=z_{c}$ instead of $z=z_{I R}$. Therefore, we will summarize the results for the meson spectra in the braneless approach omitting the calculational details.

From Eq. (12), we see that the vector meson mass depends on the position of the singularity only. So we can find the position of $z_{c}$, where the mass of the first excited mode $m_{\rho}$ is assigned the experimentally known value $776 \mathrm{MeV}$. By choosing the boundary conditions, $V_{i}^{(1)}=0$ at $z=0$ and $\partial_{z} V_{i}^{(1)}=0$ at $z=z_{c}$, the position of the singular point is fixed as $z_{c}=1 / 325 \mathrm{MeV}$. Inserting this value into Eq. (5), the value of the gluon condensation for $N_{c}=3$ is obtained as

$$
\left\langle\operatorname{Tr} G^{2}\right\rangle \approx 0.139\left[\mathrm{GeV}^{4}\right]
$$

It is larger than the the commonly expected value, $0.012\left[\mathrm{GeV}^{4}\right][25]$. Moreover, as it is known in the lattice QCD that the gluon condensation in the confining phase decreases as the temperature increases, it is interesting to investigate the meson masses varying gluon condensation. In Figure 


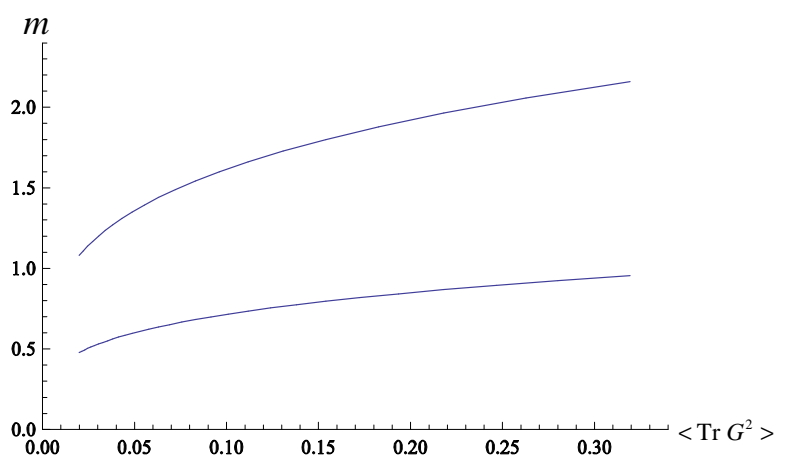

Figure 2: $\rho$-meson masses in GeV unit of the first and second modes, $V_{i}^{(1)}$ and $V_{i}^{(2)}$, depending on the gluon condensation in the braneless approach.

2 we show the first and second excited vector meson masses. Unlike the case of the hard wall approach, the vector meson masses strongly depend on the gluon condensation. Moreover, the vector meson masses increase as the gluon condensation increases, which is opposite to the hard wall case.

The scalar field $v$ in the braneless approach has a $\log$ divergence at $z=z_{c}$ as shown in Eq. (18). Fortunately, the masses for the axial vector and pseudoscalar meson in Eq. (20) depend only on $\Gamma(z)$, the combination of the above logarithmic divergence and the metric component. With the help of the metric component, which vanishes at $z=z_{c}, \Gamma(z)$ also becomes zero. As a result, in all equations for the axial vector and pseudoscalar meson the divergent terms do not appear. Imposing the boundary conditions, $\varphi=0, \pi=0$ at $z=0$ and $\partial_{z} \varphi=0$ at $z=z_{c}$, we find the masses of the axial vector and pseudoscalar meson depending on the gluon condensation as drawn in Figure 3. For numerically exact values, see Table 2.

\begin{tabular}{|c||c|c|c|c|}
\hline$z_{c}(1 / \mathrm{GeV})$ & $\left\langle\operatorname{Tr} G^{2}\right\rangle\left(\mathrm{GeV}^{4}\right)$ & $m_{\rho}(\mathrm{GeV})$ & $m_{A}(\mathrm{GeV})$ & $m_{\pi}(\mathrm{GeV})$ \\
\hline \hline $1 / 0.200$ & 0.020 & 0.4780 & 1.4081 & 0.13796 \\
\hline $1 / 0.250$ & 0.049 & 0.5975 & 1.4057 & 0.13808 \\
\hline $1 / 0.325$ & 0.139 & 0.7768 & 1.3574 & 0.14020 \\
\hline $1 / 0.378$ & 0.253 & 0.9035 & 1.2880 & 0.14743 \\
\hline $1 / 0.400$ & 0.319 & 0.9561 & 1.2715 & 0.15302 \\
\hline
\end{tabular}

Table 2 : Meson masses depending on the gluon condensation in the braneless approach.

One can see from the Table 2 and the figures that the gluon condensation effect on the meson masses in the braneless approach is not small unlike the cases of the hard wall approach. Furthermore, the masses of the vector and axial vector meson in the braneless approach qualitatively behave opposite to those in the hard wall approach.

In the case of the dissociation of the quarkonium and the monopole-anti monopole bound state, the distance between the quark and anti-quark or the monopole and anti-monopole is described by the same integral equations, Eq. (28) or Eq. (234), respectively. The free energy 


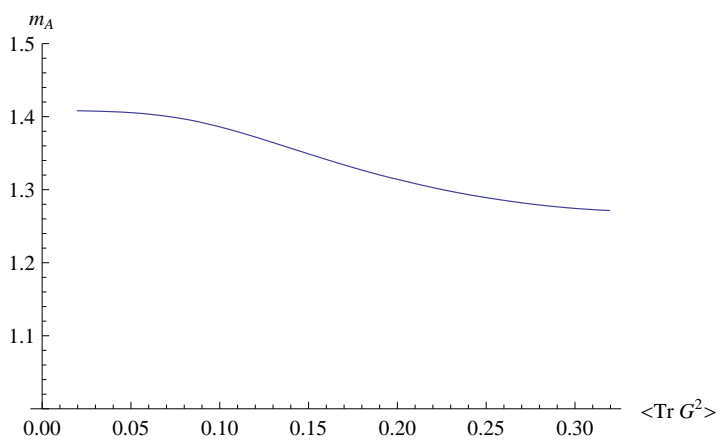

(a)

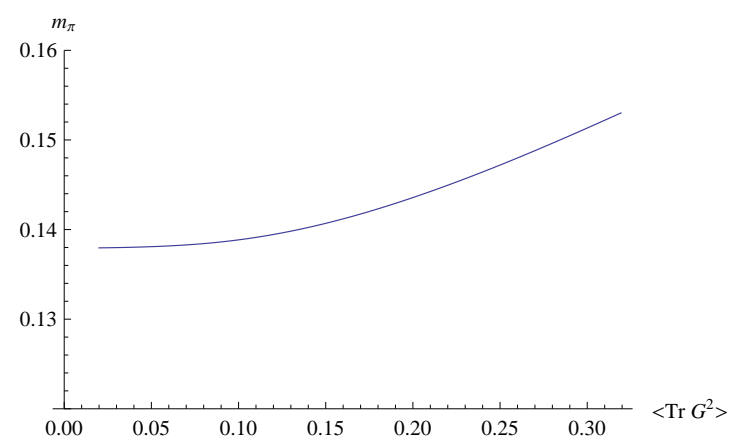

(b)

Figure 3: (a) axial vector meson mass and (b) pion mass in the unit of $\mathrm{GeV}$ depending on the gluon condensate in the braneless approach.

differences for quarkonium and monopole-anti monopole bound state are slightly changed into

$$
\begin{aligned}
V_{F 1}= & 2 \int_{0}^{z_{0}} d z \frac{\Delta^{2}}{z^{2} \Delta_{0}} \frac{\left(1-c^{2} z^{8}\right)^{3 / 4}}{\sqrt{\frac{\Delta^{2}}{\Delta_{0}^{2}}\left(1-c^{2} z^{8}\right)-\frac{z^{4}}{z_{0}^{4}}\left(1-c^{2} z_{0}^{8}\right)}} \\
& -2 \int_{0}^{z_{c}} d z \frac{\Delta\left(1-c^{2} z^{8}\right)^{1 / 4}}{z^{2}}, \\
V_{D 1}= & 2 \int_{0}^{z_{0}} d z \frac{\Delta_{0}}{z^{2} \Delta^{2}} \frac{\left(1-c^{2} z^{8}\right)^{3 / 4}}{\sqrt{\frac{\Delta_{0}^{2}}{\Delta^{2}}\left(1-c^{2} z^{8}\right)-\frac{z^{4}}{z_{0}^{4}}\left(1-c^{2} z_{0}^{8}\right)}} \\
& -2 \int_{0}^{z_{c}} d z \frac{\left(1-c^{2} z^{8}\right)^{1 / 4}}{z^{2} \Delta} .
\end{aligned}
$$

From Eq. (28), Eq. (34) and Eq. (37), the dissociation lengths of the quarkonium and monopole-anti monopole bound state are represented in Figure 5 (a) and (b). Unlike the hard wall approach case, the dissociation of the quarkonium and monopole-anti monopole bound state decreases as the gluon condensation increases.

\section{Summary and Discussion}

We have studied meson spectra on the gluon condensate background using two alternative approaches, termed "hard wall" and "braneless", in the holographic QCD model. The backreaction of the scalar field corresponding to the gluon condensation usually causes a divergence, which gives a naked singularity in the bulk geometry.

In the hard wall model, we introduced an IR cutoff by hand in order to describe the confining phase and assumed that an IR cutoff exists "in front" of the singularity. In this case, because the IR cutoff screens the singularity, we do not need to worry about the existence of the singularity. If the IR cutoff lies behind the singularity, the results become those of the braneless approach. We found that the quantitative change of meson masses does not strongly depend on the gluon 


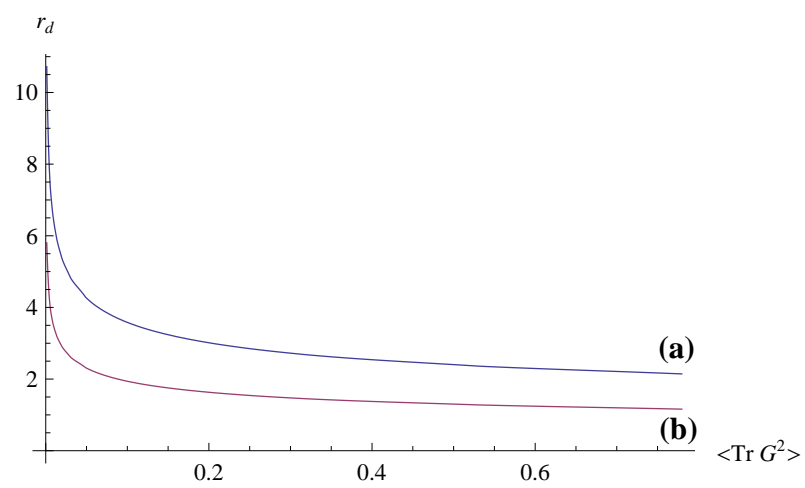

Figure 4: Dissociation length, $r_{d}$ in the unit of $\mathrm{GeV}^{-1}$, depending on the gluon condensation in the case of (a) quarkonium and (b) the bound state of monopole-anti monopole in the braneless approach.

condensate in the hard wall approach. Qualitatively, the vector meson mass decreases slightly as the gluon condensation increases. On the contrary, masses of the axial vector meson and pion increase very slowly. Furthermore, we also found that the dissociation length of the quarkonium and monopole anti-monopole bound state becomes respectively larger and shorter, as the gluon condensation increases.

In the braneless approach, where the singularity was identified with the IR cutoff, we have shown that the equations for meson spectra are well defined in spite of the singularity. Due to the merit of the braneless approach, in which we do not need to introduce an IR cutoff by hand, we also investigated the meson spectra. In this approach, we found that meson masses similar to those in the EKSS model [5] can be obtained for $\left\langle\operatorname{Tr} G^{2}\right\rangle=0.139[\mathrm{GeV}]^{4}$, which is bigger than the commonly expected value of the gluon condensation, $0.012[\mathrm{GeV}]^{4}$. Moreover, we found that the meson spectra significantly depend on the gluon condensate and that masses of the vector meson and pion increase as the gluon condensation becomes large, while the axial vector meson mass decreases. For the dissociation of the quarkonium and monopole anti-monopole bound state it was shown that the dissociation length of both increases as the gluon condensate increases. This implies that it gets more difficult to dissociate a quarkonium and monopole anti-monopole bound states for a large value of the gluon condensate.

The two alternative approaches considered here give totally different results for the meson masses. As we expect that the gluon condensate may not affect the meson spectra strongly, the hard wall model might be more appropriate to describe the gluon condensate than the braneless approach. To clarify this further, it would be interesting to compare our results with observations in future experiments. Our work might be helpful for understanding the gluon condensation effect in real QCD.

\section{Acknowledgement}


This work was supported by the National Research Foundation of Korea(NRF) grant funded by the Korea government(MEST) through the Center for Quantum Spacetime(CQUeST) of Sogang University with grant number 2005-0049409."

\section{References}

[1] J. M. Maldacena, Adv. Theor. Math. Phys. 2, 231 (1998) [Int. J. Theor. Phys. 38, 1113 (1999)] arXiv:hep-th/9711200]; S. S. Gubser, I. R. Klebanov and A. M. Polyakov, Phys. Lett. B 428, 105 (1998) arXiv:hep-th/9802109]; E. Witten, Adv. Theor. Math. Phys. 2, 253 (1998) arXiv:hep-th/9802150]; O. Aharony, S. S. Gubser, J. M. Maldacena, H. Ooguri and Y. Oz, Phys. Rept. 323, 183 (2000) arXiv:hep-th/9905111; J. Erdmenger, N. Evans, I. Kirsch and E. Threlfall, Eur. Phys. J. A 35, 81 (2008) arXiv:0711.4467 [hep-th]].

[2] G. Policastro, D. T. Son and A. O. Starinets, JHEP 0209, 043 (2002) arXiv:hep-th/0205052; G. Policastro, D. T. Son and A. O. Starinets, JHEP 0212, 054 (2002) arXiv:hep-th/0210220.

[3] C. P. Herzog, Phys. Rev. Lett. 98, 091601 (2007) arXiv:hep-th/0608151.

[4] T. Sakai and S. Sugimoto, Prog. Theor. Phys. 113, 843 (2005) arXiv:hep-th/0412141.

[5] J. Erlich, E. Katz, D. T. Son and M. A. Stephanov, Phys. Rev. Lett. 95, 261602 (2005) arXiv:hep-ph/0501128.

[6] A. Karch, E. Katz, D. T. Son and M. A. Stephanov, Phys. Rev. D 74, 015005 (2006) arXiv:hep-ph/0602229.

[7] L. Da Rold and A. Pomarol, Nucl. Phys. B 721, 79 (2005) arXiv:hep-ph/0501218.

[8] S. J. Sin and Y. Zhou, JHEP 0905, 044 (2009) arXiv:0904.4249 [hep-th]]; S. J. Sin, S. Yang and Y. Zhou, JHEP 0911, 001 (2009) arXiv:0907.1732 [hep-th]].

[9] U. Gursoy, E. Kiritsis, L. Mazzanti and F. Nitti, JHEP 0905, 033 (2009) arXiv:0812.0792 [hep-th]].

[10] J. Babington, J. Erdmenger, N. J. Evans, Z. Guralnik and I. Kirsch, Phys. Rev. D 69, 066007 (2004) arXiv:hep-th/0306018.

[11] N. J. Evans and J. P. Shock, Phys. Rev. D 70, 046002 (2004) arXiv:hep-th/0403279].

[12] M. Kruczenski, D. Mateos, R. C. Myers and D. J. Winters, JHEP 0405, 041 (2004) arXiv:hep-th/0311270. 
[13] B. H. Lee, C. Park and S. J. Sin, JHEP 0907, 087 (2009) arXiv:0905.2800 [hep-th]].

[14] C. Park, arXiv:0907.0064 [hep-ph].

[15] K. Jo, B. H. Lee, C. Park and S. J. Sin, arXiv:0909.3914 [hep-ph].

[16] H. Boschi-Filho and N. R. F. Braga, Eur. Phys. J. C 32, 529 (2004) arXiv:hep-th/0209080;

H. Boschi-Filho and N. R. F. Braga, JHEP 0305, 009 (2003) arXiv:hep-th/0212207];

H. Boschi-Filho, N. R. F. Braga and C. N. Ferreira, Phys. Rev. D 74, 086001 (2006) arXiv:hep-th/0607038; C. A. Ballon Bayona, H. Boschi-Filho, N. R. F. Braga and L. A. Pando Zayas, Phys. Rev. D 77, 046002 (2008) [arXiv:0705.1529 [hep-th]].

[17] Y. Kim, B. H. Lee, S. Nam, C. Park and S. J. Sin, Phys. Rev. D 76, 086003 (2007) arXiv:0706.2525 [hep-ph]].

[18] J. P. Shock, F. Wu, Y. L. Wu and Z. F. Xie, JHEP 0703, 064 (2007) arXiv:hep-ph/0611227]; Y. L. Wu and Z. F. Xie, JHEP 0710, 009 (2007) arXiv:0705.2360 [hep-ph]].

[19] S. S. Gubser, arXiv:hep-th/9902155.

[20] Y. Kim, B. H. Lee, C. Park and S. J. Sin, JHEP 0709, 105 (2007) arXiv:hep-th/0702131.

[21] A. Kehagias and K. Sfetsos, Phys. Lett. B 454, 270 (1999) arXiv:hep-th/9902125; C. Csaki, J. Erlich, C. Grojean and T. J. Hollowood, Nucl. Phys. B 584, 359 (2000) arXiv:hep-th/0004133; S. Nojiri and S. D. Odintsov, Phys. Rev. D 61, 024027 (2000) arXiv:hep-th/9906216; D. Bak, M. Gutperle, S. Hirano and N. Ohta, Phys. Rev. D 70, 086004 (2004) arXiv:hep-th/0403249.

[22] C. Csaki and M. Reece, JHEP 0705, 062 (2007) arXiv:hep-ph/0608266.

[23] N. Evans, A. Tedder and T. Waterson, JHEP 0701, 058 (2007) arXiv:hep-ph/0603249.

[24] Y. Kim, B. H. Lee, C. Park and S. J. Sin, Phys. Rev. D 80, 105016 (2009) arXiv:0808.1143 [hep-th]].

[25] D. E. Miller, Phys. Rept. 443, 55 (2007) arXiv:hep-ph/0608234.

[26] J. A. Minahan, Adv. Theor. Math. Phys. 2, 559 (1998) arXiv:hep-th/9803111.

[27] N. R. Constable and R. C. Myers, JHEP 9911, 020 (1999) arXiv:hep-th/9905081. 